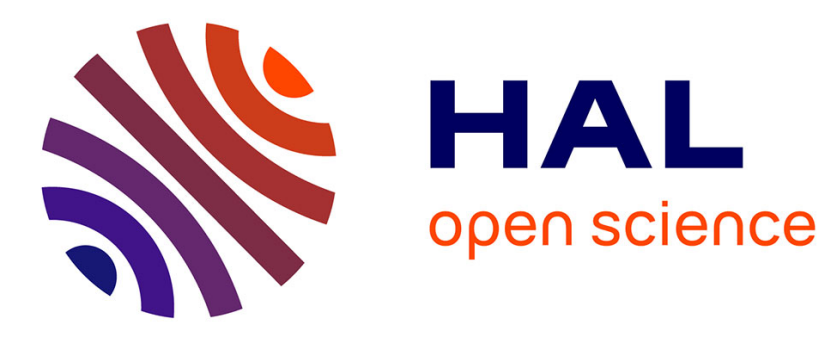

\title{
Acceptabilité des inégalités et exigence de justification
}

Caroline Guibet Lafaye, Emmanuel Bernard Picavet

\section{To cite this version:}

Caroline Guibet Lafaye, Emmanuel Bernard Picavet. Acceptabilité des inégalités et exigence de justification. Filosofia Unisinos - Unisinos Journal of Philosophy, 2010, 11 (1), p. 2-16. hal-00384838

\section{HAL Id: hal-00384838 \\ https://hal.science/hal-00384838}

Submitted on 15 May 2009

HAL is a multi-disciplinary open access archive for the deposit and dissemination of scientific research documents, whether they are published or not. The documents may come from teaching and research institutions in France or abroad, or from public or private research centers.
L'archive ouverte pluridisciplinaire HAL, est destinée au dépôt et à la diffusion de documents scientifiques de niveau recherche, publiés ou non, émanant des établissements d'enseignement et de recherche français ou étrangers, des laboratoires publics ou privés. 
Caroline Guibet Lafaye et Emmanuel Picavet ${ }^{1}$

\section{« L’acceptabilité des inégalités »}

Universités européennes d'été 2006.

Neuvième université d'été d'histoire de la pensée et méthodologie économiques.

« Répartition et croissance »; Lyon, Université Lumière (Lyon II), 2-6 septembre 2006.

\section{Introduction}

Dans plusieurs pays, le discours politique et économique courant exprime une forte présomption en faveur de l'égalité. Cette présomption caractérise également un certain discours philosophique, en ce sens qu'aucune raison ne semble requise pour justifier une répartition égale des avantages : celle-ci se présente alternativement comme « naturelle » ou comme juste et bonne en soi. Elle ne nécessite aucune justification dans la mesure où elle est conçue comme se justifiant par elle-même. Ainsi, on postule que l'égalité n'appelle aucun commentaire et que seule l'inégalité doit être justifiée ; qu'il n'y a pas à rendre compte de l'uniformité, de la régularité, de la similitude, de la symétrie, tandis que les différences exigent une explication et, en règle générale, une justification ${ }^{2}$.

La présomption en faveur de l'égalité signifie que les différences de traitement demandent à être justifiées et qu'une entorse à l'égalité n'est possible qu'en vertu d'une raison particulière. Lorsque, par exemple, je partage un gâteau en dix parts égales pour les distribuer à dix personnes, en en donnant exactement un dixième à chacune, on n'exige pas de justification. En revanche, si je m'écarte de ce principe de la division à parts égales, on attend de moi que j'avance une raison

1 Cette collaboration s'inscrit dans le cadre de notre participation conjointe au programme DELICOM de l'Université Panthéon-Sorbonne (ANR- JC 05) et au réseau “Applied Global Justice" (Research training network, Commission européenne). Nous remercions ces institutions pour leur appui. Nous adressons aussi nos remerciements à Christian Arnsperger, Paul Cobben, Luc Foisneau, Peter Köller, Urs Marti, Jean-Christophe Merle et Hermann van Erp, pour de nombreuses discussions autour de ces enjeux au cours des dernières années.

${ }^{2}$ Voir Isaiah Berlin, «Equality », repr. in Frederick A. Olafson (éd.), Justice and Social Policy, Englewood Cliffs, NJ, Prentice-Hall, 1961, p. 131. 
particulière ou une procédure de choix faisant apparaître comme rationnelle la configuration choisie. Mais cela peut-il être étendu à l'échelle d'une société entière

A tout le moins, il faut reconnaître qu'il existe vraisemblablement différents types de jugement motivés par l'inégalité, et qu'il y a lieu de chercher à identifier ceux qui comportent des enjeux éthiques réels. Nous pensons que cette recherche coïncide largement avec l'étude de l'acceptabilité relative, par les agents sociaux, des différentes sortes d'inégalité.

Ce type d'enquête est également appelé par la suspicion d'une variabilité culturelle des modes d'expression du traitement égal. L'égalité est souvent rapportée à l'expression d'un égal respect, mais les manières de manifester du respect à autrui sont a priori dépendantes de la culture qui prévaut. Par exemple, pratiquer la discrimination positive aux Etats-Unis est souvent perçu comme une manière légitime de réparer un tort ou une inégalité des chances dans la vie, alors qu'en France, les mêmes actions sont repoussées au motif qu'elles traduiraient une inégalité de traitement, traduisant une reconnaissance de l'inégalité des citoyens, voire l'existence de plusieurs communautés au sein de la Nation. On pourrait se poser des questions analogues concernant la jouissance des biens et des revenus ainsi que les diverses formes de réussite sociale. Philosophiquement il n'est pas évident que montrer qu'un égal respect à tous doive se traduire par le souci actif de la réussite sociale des uns et des autres, même s'il est possible que certaines particularités culturelles y conduisent. Le même respect pourrait se traduire d'une autre manière, par exemple, en s'exprimant par des préceptes tels que «que chacun se débrouille », «que chacun assume les conséquences de ses choix dans la vie...», " que chacun se soumette aux règles impartiales du marché », etc.

La thématique de l'égalité mobilise des intuitions et des raisons morales mais pose aussi question, du point de vue des modalités pratiques de concrétisation politique et administrative, et du point de vue de l'évolution du débat public. Par exemple, on peut se poser les questions suivantes: est-ce que la mise en œuvre de principes d'égalité comporte des désavantages notables ? Est-ce qu'elle ne favorise pas à l'occasion la dissimulation de vrais motifs par des raisons seulement apparentes?

Ce sont là autant de motivations justifiant de ne pas s'en tenir avec trop d'assurance au point de vue d'après lequel toute forme de mise en question de l'exigence d'égalité doit être a priori disqualifiée comme une forme ou une autre de rhétorique réactionnaire. On prendra ici le parti de 
considérer qu'il y a peut-être à s'instruire de la critique (à un certain niveau au moins) des idéaux d'égalité.

\section{Toutes les inégalités sont-elles dommageables?}

1.1 Le coût de la correction des inégalités.

Chez des auteurs tels que Michael Polanyi (Logique de la liberté) et Robert Nozick (Anarchie, Etat, Utopie), on trouve des arguments hyperboliques, qui s'inscrivent dans une certaine tradition du libéralisme politique et qui mettent l'accent sur le potentiel liberticide des idéaux sociaux (à l’opposé de tendances progressistes au sein de la famille libérale).

Or, il y aurait lieu de considérer aussi des coûts plus modérés : des inconvénients sociaux, qui ne vont pas nécessairement jusqu'à des pertes de liberté caractérisées. Par exemple, le système des HLM a certainement comporté des réussites, dans la promotion de l'accès équitable au logement ; mais il a favorisé aussi des effets perçus de ségrégation spatiale de différentes parties de la population (avec l'émergence de catégories du discours courant telles que «jeunes des banlieues », « quartier populaire » [dans le nouveau sens de l'expression : non pas le faubourg animé, mais une réalité plus inquiétante]...).

Désormais, les efforts concrets pour corriger les inégalités se traduisent à l'occasion par des tentatives d'application de principes controversés qui, aux yeux des uns ou des autres, comportent des désavantages. En l'occurrence, le principe de "mixité sociale (mélanger les cultures, mélanger les statuts sociaux). Officiellement, ce principe est un principe de réalisation de l'égalité (ne pas enfermer les personnes, qui ont des problèmes dans des ghettos sociaux, donner à chacun les mêmes chances...).

Or l'application de ce principe, sans être en rien « liberticide», donne lieu à des désavantages perçus qui semblent bien avoir une certaine réalité, tels que (1) l'aggravation de la situation du logement pour des familles très modestes; (2) le risque de stigmatisation sociale accrue, par constitution de catégories d'administrés dans un pays où, par tradition, les citoyens ne sont pas répartis en groupes hétérogènes; (3) le traitement différencié des citoyens, selon la zone de résidence ou de demande de résidence (ce qui entretient un rapport inconfortable avec la tradition républicaine de la banalité du territoire). Ce sont là des inconvénients politiques qui ne vont 
certainement pas jusqu'à la remise en cause des libertés, mais qui n'en sont pas moins réels. Ces inconvénients ne sont d'ailleurs pas forcément suffisants pour contrebalancer d'éventuels avantages: les reconnaître ne suffit donc pas à condamner ce type de politique. Plus généralement, il faut simplement rappeler que les politiques de correction des inégalités peuvent présenter divers inconvénients. Pour cette raison, le maintien de certaines inégalités peut ne pas être dommageable, tout bien pesé.

\subsection{La vie sociale suppose-t-elle une répartition des rôles qui passe par des inégalités?}

D'un point de vue moral, les contingences naturelles, attachées aux conditions dans lesquelles les individus sont nés, sont aussi arbitraires que l'identité de leur famille, de leur voisinage, de leur nation. Comme on le sait, la théorie morale propose un répertoire d'expériences de pensée (voile d'ignorance, observateur impartial, situation de dialogue idéale), destinées à annuler les raisons contingentes de traiter comme des données, des particularités dans la position des individus, qui ont une source seulement contingente. Néanmoins, d'un point de vue politique, le risque est d'écarter trop vite des complémentarités entre des choix de vie, qui présentent des avantages collectifs, pour la seule raison qu'on y percevrait des inégalités contestables parce qu'arbitraires.

Cela peut passer pour un argument réactionnaire et nul doute que ce ne soit effectivement le cas, dans d'assez nombreuses occurrences. Mais l'argument renvoie aussi à une réalité à laquelle il faut faire face : en l'absence de bouleversements sociaux de très grande ampleur, qui pourraient présenter un coût ou des risques prohibitifs, il est de fait que certains avantages notables vont de pair, empiriquement, avec des désavantages (ou des choix de vie particuliers), qui sont également notables.

La problématique des capacités (capabilities), développée par A. Sen présente l'intérêt de ménager une place aux choix de vie. Toutefois, A. Sen ne remet pas en question le postulat vraisemblablement faux selon lequel «plus les gens ont des choix (ou des opportunités), mieux c'est ». Or, le fait d'avoir plus de choix ou de capacité de choisir va empiriquement avec des styles de vie que tout le monde ne désire pas forcément.

Par exemple, la jouissance d'une grande et belle demeure (ou d'un beau navire...) va de pair avec l'obligation précise de gérer ou d'administrer ce bien, même si l'on fait abstraction de la 
fiscalité. Cela prend du temps et doit aller de pair, dans la plupart des cas, avec des choix de vie particuliers. Il en résulte ordinairement des avantages qui bénéficient largement à la population (entretien de parcs et de paysages, capacité à influer sur les pouvoirs publics via des associations de riverains, pour assurer la qualité de l'environnement, face aux lobbies poussant les pouvoirs publics en sens contraire). Par ailleurs, certaines catégories de rémunération tendent à être réservées à des dirigeants d'entreprise ou à des personnalités du sport ou du spectacle, dont le mode de vie habituel, comme cela est bien connu, comporte des servitudes très particulières.

Lorsqu'on raisonne sur le mode normatif en termes d'égalité, on se concentre habituellement sur certains avantages comme la rémunération. Or il est manifestement difficile d'opérer un découplage entre ce type d'élément et des aspects corrélés du mode de vie - ce dont on tient compte, d'ailleurs, lorsqu'on formule des critères du type «absence d'envie » en économie. En effet, le concept de non-envie répond à une idée intuitive d'acceptabilité pour chacun, compte tenu de la situation des autres. Ainsi, on tient compte de la vie sociale sans avoir à considérer toutes les allocations possibles. Le critère est satisfait lorsqu'aucune personne n'en envie une autre ou lorsqu'aucune paire d'individus ne se trouve dans cette situation. Selon ce critère, plus précisément, aucun individu ne doit préférer à la combinaison de biens, qui lui est allouée, la combinaison de biens, qui est allouée à un autre individu ${ }^{3}$. Cela peut donner une raison de considérer que toutes les inégalités ne sont pas, par principe, inacceptables. Or, c'est justement bien l'acceptabilité, qui est ici directement en cause : on se demande, en effet, si la situation peut être jugée acceptable par un individu, qui pourrait être tenté de remettre en cause l'ordre des choses, dès lors qu'il estime la situation d'un tiers préférable à la sienne propre. L'équité n'intervient dans ce contexte que si l'on choisit pour ce deuxième concept une interprétation très particulière, qui privilégie la réaction de chaque individu à la situation des autres.

Par ailleurs, les différences de mode de vie sont corrélées avec des spécialisations sociales dans certaines tâches, et il est vrai que cette spécialisation peut comporter des avantages largement partagés. Se pose alors la question de l'aptitude de la société à exploiter, au profit de chacun, les complémentarités qui en résultent, grâce à des incitations (ou récompenses) appropriées. Cela peut passer par l'acceptation d'inégalités, ce que résume le «principe de

\footnotetext{
${ }^{3}$ Ce critère peut être utilisé dans des situations multidimensionnelles, où l'on cherche à construire des critères d'équité, fondés sur des caractéristiques concrètes des allocations.
} 
différence ${ }^{4}$. Ce qui est injustifiable en termes de mérite (car ce que l'on appelle le mérite est déterminé de part en part par des caractéristiques contingentes de la société) peut cependant être justifié en référence à l'efficacité. Dans la théorie rawlsienne de la justice, l'inégalité peut résulter du second principe de justice, qui régit la structure des organisations et la répartition, de manière à ce que la coopération sociale soit à la fois juste et efficace ${ }^{5}$.

La référence au principe de différence est alors décisive, dans la justification d'une inégalité ou d'une entorse à l'égale distribution des parts, en ce sens que l'inégalité est acceptable, dans la mesure où elle sert à améliorer la position des plus mal lotis. La reconnaissance du principe de différence redéfinit les raisons, justifiant que des inégalités sociales existent. Dans cette perspective et dans un discours où l'égalité est la règle, les différences entre personnes sont arbitraires, d'un point de vue moral, si aucun argument moral ne permet de conclure qu'il doit y avoir des différences. Autrement dit, il y a une présomption contre certaines différences que seules des raisons morales permettraient d'outrepasser (ou de neutraliser) ${ }^{6}$.

Le principe libéral de la juste (fair) égalité des chances nuance ces perspectives en refusant l'idée que l'on puisse améliorer la situation de chacun, en attribuant à certaines positions des pouvoirs et des avantages, alors même que certains groupes en sont exclus. C'est le principe libéral de l'ouverture à tous des positions sociales. Ce principe exprime la conviction que si certaines places n'étaient pas ouvertes à tous, sur une base équitable, ceux qui en seraient exclus auraient raison de s'estimer injustement traités, même s'ils bénéficiaient de cet inégal accès aux fonctions sociales, comme le prétendent certains dans certains contextes ${ }^{7}$.

\footnotetext{
${ }^{4}$ J. Rawls, Théorie de la justice [1971], Paris, Seuil, 1987, p. 138. Voici une formulation simple du principe : «Les inégalités sociales et économiques doivent être organisées de façon à ce que, à la fois, (a) elles apportent aux plus désavantagés les meilleures perspectives et (b) elles soient attachées à des fonctions et à des positions ouvertes à tous, conformément à la juste (fair) égalité des chances. » (Théorie de la justice, p. 115).

${ }^{5}$ J. Rawls, Théorie de la justice, Paris, Seuil, 1987, p. 549.

${ }^{6}$ On peut se demander si ce principe est un précepte méthodologique commode (un «principe de raison insuffisante »), en éthique, ou bien un véritable principe d'éthique substantielle. Le commentaire est un peu plus développé à ce propos dans : E. Picavet, John Rawls, Théorie de la justice, I (Paris, Ellipses, 2001).

${ }^{7}$ Voir J. Rawls, Théorie de la justice, p. 116. Chacune de ces personnes pourrait se plaindre d'avoir été détournée de l'expérience de la réalisation de soi-même et, par conséquent, privée de l'une des principales formes du bien pour les êtres humains. Parmi les auteurs qui ont défendu le caractère avantageux de l'inégal accès aux positions sociales, citons Michael Oakeshott, qui a longuement argumenté en faveur de l'accès inégal aux positions de pouvoir («Le rationalisme en politique », tr. fr. Olivier Sedeyn, Cités, 14 [2003], p. 121-157, initialement dans Cambridge Journal, I [1947]). S'en prenant au « rationalisme», dont il cite quelques « rejetons » (l'Education Act de 1944, le Vote des femmes, le projet d'un fonctionnariat basé sur nulle autre qualification que les capacités personnelles - p. 127), Oakeshott se faisait fort d'éclairer les intentions profondes de l'exposé d'une technique du politique chez Machiavel : "Le gouvernant bien établi, éduqué dans une tradition et héritier d'une longue expérience familiale, semblait suffisamment bien équipé pour la position qu'il occupait ; [...] en général il savait comment se conduire.
} 
Si la spécialisation des formes de vie est prise au sérieux, dans le domaine de la division du travail ou sous le rapport d'une structure incitative contrastée, justifiée par des considérations d'efficacité, on ne peut en dire autant des aspects de cette spécialisation qui concernent la consommation, la jouissance ou la possession des biens. Or, est-il si absurde d'admettre que la consommation des uns bénéficie aux autres dans certains cas? Cela est relativement bien admis dans le cas des biens éducatifs (si la fréquentation d'un certain cursus associé à certains avantages a des effets positifs pour la société).

Mais allons plus loin : il n'y a pas de vraie raison de dévaloriser a priori ou de rattacher systématiquement à des phénomènes de fausse conscience (ou d'existence par procuration) l'intérêt que peuvent trouver des individus, moins favorisés, à des formes de vie plus favorisées. Par exemple, on peut prendre intérêt à la lutherie de haut niveau ou au sport automobile, tout en étant très loin de pouvoir se procurer soi-même les objets du désir. Il n'est pas absurde de considérer que ces «objets du désir » que sont les très belles voitures ou les très bons violons traduisent parfois des formes d'excellence ou d'accomplissement (dans les techniques, l'artisanat ou les applications de la science), qui s'inscrivent dans un progrès commun ou dans l'histoire de l'humanité, et qui peuvent souvent donner lieu à des formes de partage, sans qu'il y ait pour autant égalité des possessions.

Par exemple, seule une personne très riche peut se procurer un Stradivarius mais si cette personne confie l'objet à un(e) violoniste, les bénéfices peuvent en être partagés avec un public large. Peu de gens peuvent acheter un Amati, mais de nombreuses personnes peuvent se procurer un violon «de la famille Amati» (une copie française ou allemande correcte - on ne parle d'ailleurs pas de « faux » à cet égard car il ne s'agit pas de tromper sur la nature du bien). Dans le monde contemporain, d'une manière beaucoup plus générale, l'accès aux biens de luxe - par exemple un beau stylo ou un petit vase en cristal - est une chose très largement partagée. Et les biens qui sont typiquement associés à des formes d'inégalité sociale servent souvent d'exemples ou de modèles, pour des produits plus largement accessibles. Il y a par là des formes de bénéfice

Mais il en était tout autrement pour le gouvernant nouveau, qui n'apportait à sa fonction que les qualités qui lui avaient permis d'obtenir le pouvoir politique et qui n'avait rien appris facilement sinon les vices liés à sa fonction, le caprice du prince. » (pp. 145-146). La critique est plus dure encore concernant Marx et Engels, « les auteurs du plus extraordinaire de nos rationalismes politiques, comme on pouvait bien s'y attendre, car il a été composé pour l'instruction d'une classe moins éduquée politiquement que toute autre qui ait jamais pu nourrir l'illusion d'exercer un pouvoir politique» (p. 147). Le niveau de conduite qui est «le propre d'une grande profession » est supposé transmissible par « une tradition familiale» (p. 155). 
commun à tirer de biens inégalement répartis et qui peuvent par ailleurs faire l'objet d'envie. En somme, l'envie (ou le désir de susciter l'envie) n'est pas le dernier mot : dans la réalité, le partage des valeurs prend des formes plus complexes.

\subsection{Quel bénéfice attendre de la correction des inégalités?}

La justification de l'inégalité est en fait aussi le point d'appui principal de la critique adressée au principe de différence de Rawls, par les auteurs qui se veulent « plus égalitaristes » que Rawls, tels Gerry Cohen, John Roemer, Marc Fleurbaey. La division sociale des rôles selon Rawls suppose qu'il y ait des désavantages mais l'auteur ne s'intéressait guère à la correction, en pratique, des sources contingentes de répartition biaisée des rôles - du moins, pas au-delà de considérations très générales sur la réalisation d'une valeur équitable de la juste égalité des chances.

Néanmoins l'ambition de corriger les inégalités, qui sont dues au hasard, reconduit nécessairement à la question des raisons, appelant une «correction », et donc, à la question du type de dommage associé aux inégalités en tant que telles. Il y a autour de ces questions des désaccords considérables.

Par exemple, les contributions de Ronald Dworkin ont attiré l'attention sur le fait que toute inégalité n'était pas obligatoirement à considérer comme un problème en soi (ou du moins, un problème moral). En particulier, Dworkin soulignait que les inégalités ne sont souvent que le reflet inévitable de choix de vie contrastés et également légitimes. Or, on le sait, cette ligne d'argumentation était solidaire de la défense d'une certaine forme d'égalité : l'égalité des ressources, devant permettre aux individus de faire des choix de vie également significatifs. On voit, sur cet exemple, que les idéaux de correction des aspects arbitraires de l'inégalité ne reposent pas nécessairement sur l'idée que toute inégalité est, par elle-même, une mauvaise chose ou un problème.

Revenons justement à cette question centrale : est-ce que toute inégalité est en elle-même problématique ? Considérons une comparaison entre une situation (I) et une situation (II) :

- (I) deux individus constitutifs d'une société ont un niveau de vie médiocre et semblable ;

- (II) ces deux individus ont des niveaux contrastés, mais chacun a un niveau de vie plus élevé que dans la situation (I). 
Y a-t-il alors des raisons de préférer (I) à (II) ?

Un égalitariste attaché à l'égalité par principe pourrait faire valoir que (II) a l'inconvénient d'être une situation inégalitaire, en sorte que la transition de (I) vers (II) est marquée par un regrettable développement de l'inégalité.

Toutefois la portée de cette observation doit s'apprécier en rapport avec le caractère plus ou moins «inclusif» de l'indicateur de niveau de vie. (a) Si l'indicateur est suffisamment « inclusif », pour incorporer des inconvénients spécifiques, naissant d'un degré d'inégalité dans la société, l'argument a une portée faible, voire nulle : en effet, la position du plus défavorisé, qui se trouve relevée dans la transition de (I) à (II), tient déjà compte de ces inconvénients spécifiques; on ne voit pas alors pourquoi il faudrait en tenir compte deux fois. Sa position s'améliore « tout bien pesé », en tenant compte des désavantages de l'inégalité.

(b) Si par contre l'indicateur se concentre seulement sur des données partielles (disons, le niveau de revenu), en oubliant d'autres dimensions objectives du bien-être, qui risquent d'être affectées par l'accroissement de l'inégalité des conditions (disons, la qualité relative ou la réputation sociale de l'éducation reçue), alors l'argument est moins convaincant. La vérité est alors que la comparaison ne livre qu'une image très partielle de l'évolution sociale.

Supposons maintenant que l'indicateur soit suffisamment « inclusif». On peut remarquer que même dans ce cas, un égalitariste pourrait certainement convaincre une partie du public : il est de fait courant de déplorer pour lui-même, et comme par principe, l'accroissement des inégalités.

Il nous semble qu'il faut alors (à défaut d'autres ressources) prendre au sérieux l'une ou l'autre des hypothèses suivantes, qui sont plus ou moins plausibles selon les cas :

$(\alpha)$ soit on a des préférences comparables aux préférences esthétiques, pour une société «plus égalitaire »; autrement dit, on « aime mieux » l'égalité. Certaines personnes admettent ainsi qu'elles « aiment » mieux vivre (ou aimeraient mieux vivre) dans une société égalitaire comme la Norvège, par comparaison avec une société inégalitaire comme les Etats-Unis ou la France. D'autres personnes apprécient les situations fortement contrastées, même si elles ne se trouvent pas personnellement du côté « avantagé » des inégalités.

( $\beta$ ) soit on considère que certains aspects de la situation personnelle comparée sont expressifs (certainement pour des raisons culturelles ou historiques) de la dignité reconnue aux personnes.

A quel degré ces considérations peuvent-elles être significatives d'un point de vue proprement éthique? Considérons chacune d'elles tour à tour. 
Le premier type de considération peut difficilement tirer sa force d'un sentiment des individus, qui serait relatif à leur propre situation (personnelle), car cela a déjà été pris en compte ex hypothesi dans l'indicateur considéré. Or cela n'a pas empêché une amélioration de la situation du plus défavorisé. La préférence pour l'égalitarisme, dont il est ici question, ne peut donc provenir de l'envie, que l'on éprouve ou que l'on n'éprouve pas, à partir de sa propre situation. Il s'agit alors vraiment, semble-t-il, d'une préférence de type esthétique. Sans s'en remettre à sa propre situation, on « aime mieux » une société égalitaire plutôt qu'inégalitaire. On peut penser que, dans ces conditions, c'est la convergence empirique des préférences, qui joue un rôle important, plutôt que l'éthique substantielle. Il peut se faire que les membres d'une communauté constatent, par un heureux hasard, la présence en eux d'aspirations convergentes, les portant à la réalisation d'une société plus égalitaire - plus «belle» (ou harmonieuse) en ce sens. Peut-être n'y a-t-il rien à y redire - pas davantage que si tous les membres d'une même famille préfèrent un concert de musique classique à un concert des Rolling Stones, - mais il ne s'agit alors ni de commandement moral ni de correction dans le jugement ou l'évaluation. En somme, il n'est pas évident du tout que l'affaire ait le moindre rapport avec l'éthique.

Le second type de considération a certainement une grande signification sociale mais d'une manière qui dépend de faits culturels contingents (à moins que la satisfaction comparée de besoins fondamentaux ou d'aspirations au développement personnel ne soient en jeu). Les considérations d'inégalité sont alors indissolublement liées à des conventions culturelles. On rencontre alors un inconvénient qui peut être réellement ressenti, mais qui est un inconvénient de l'inégalité « en conjonction avec» (des normes culturelles) plutôt que de l'inégalité « en ellemême ». On peut alors raisonner de la manière suivante : si l'inégalité est ici ressentie par les individus, qui sont liés par certaines conventions culturelles, il faut en effet s'intéresser aux sentiments de ces individus, plutôt qu'à un hypothétique inconvénient intrinsèque de l'inégalité. Et derechef, sous les hypothèses retenues, les sentiments adverses liés à l'inégalité ne sont pas de nature à annuler les effets positifs supérieurs de la configuration considérée sur la situation des moins bien lotis. 
Peut-on aller plus loin, dans la direction d'une explication des raisons pour lesquelles les conventions culturelles conduisent souvent à interpréter, en termes d'inégale manifestation de respect, les inégalités dans la situation de fortune?

Ce qu'il y a de sérieux dans l'envie ou dans la perception d'un traitement trop inégalitaire (au sens suivant : trop inégalitaire pour être compatible avec l'expression sociale d'un égal respect) a probablement à voir avec la fixité des positions sociales. Cela est suggéré par cette observation familière, que se contenter de la position sociale la plus défavorisée est relativement facile, si le passage par cette position est envisagé seulement comme une étape, dans un parcours individuel ou familial qui ne s'arrête pas là, et si la position que l'on délaissera un jour (ou que la famille délaissera un jour) est appelée à être occupée par d'autres (de jeunes travailleurs ou des travailleurs immigrés, par exemple), qui auront à leur tour les mêmes perspectives individuelles ou familiales. L'inégalité apparaît beaucoup moins facile (acceptable), si l'on a des raisons d'y voir le symptôme d'une société figée, dans laquelle des classes ou des castes coexistent de manière rigide.

Dans ce registre, on peut penser à cette forme courante d'adaptation culturelle aux inégalités qui consiste, par exemple pour les migrants, à se contenter d'un sort comparativement peu favorable avec l'espoir d'un sort meilleur (que le sien propre et que celui qui aurait été prévisible sans migration) pour les enfants. Si l'on est au-delà d'un certain seuil de conditions d'existence décentes, cette situation ne traduit pas forcément une violation moralement condamnable de l'égal traitement des personnes. Les aspirations, qui y sont associées, ne sont pas forcément à flétrir comme des formes d'adaptation psychologique à l'injustice. Après tout, il demeure vrai que la garantie de chances de réussite rigoureusement égales pour les nouveaux arrivants et pour les individus éduqués dans le pays, à supposer qu'elle soit possible, passerait à tout le moins par des changements sociaux et économiques radicaux, n'ayant aucune chance d'être validés dans les programmes des partis de pouvoir.

L'immigré de fraîche date, s'il a bénéficié de conditions d'accueil décentes (hypothèse qui n'est assurément pas toujours vérifiée !), et se voit assigner une condition sociale modeste (p.ex. un logement dans une HLM assez ordinaire et un emploi quelque peu répétitif et très moyennement rémunéré), peut en principe se tenir le raisonnement suivant: mes enfants 
bénéficient de la même éducation et des mêmes chances dans la vie que les autres, et donc les membres de ma famille (si l'on tient compte des difficultés propres à toute situation d'installation tardive dans un pays étranger) ne sont pas victimes d'inégalité de traitement. Il se peut qu'il considère que la norme culturellement sélectionnée d'égal traitement est une norme qui s'applique plutôt aux familles et aux histoires familiales qu'aux individus. Il y a de fait de nombreuses inégalités matérielles qui sont acceptées ou tolérées, sans être rapportées à une idée de traitement inégal, à cause de leur intégration dans une histoire familiale.

En résumé, il est de fait que certaines formes d'inégalité matérielle sont associées, dans certains contextes, à des sentiments d'inégale reconnaissance ou de manquement au respect égal des personnes (ex. le statut des médecins étrangers en France). Pour autant, il ne faut pas oublier la dépendance de ce fait à l'égard de formes culturelles et d'histoires individuelles ou familiales particulières. Cet argument, par ailleurs, ne peut pas justifier n'importe quelle forme d'inégalité de traitement : il en est auxquelles on ne s'adapte pas ou auxquelles on ne devrait pas s'adapter, justement parce qu'elles induisent un blocage arbitraire des perspectives individuelles ou familiales (c'est le cas vraisemblablement de toutes les formes de ségrégation sociale institutionnalisée, par exemple dans l'enseignement élémentaire ou, dans des pays développés, dans l'enseignement secondaire).

De ce point de vue, on peut remarquer que c'était la force du principe de différence de Rawls, que de s'appliquer à la configuration des positions sociales et à l'accès à ces positions, plutôt qu'au sort « instantané » de chaque individu. Le principe rawlsien de l'équité des chances permet de ne pas systématiquement tenir compte de la variété infinie des circonstances et des positions relatives et changeantes des personnes particulières ${ }^{8}$. Par là, se justifie le fait de ne pas considérer ces positions relatives comme base d'une revendication, demandant que chaque changement, envisagé comme une transaction isolée, soit juste en lui-même : «La reconnaissance des deux principes signifie donc que bien des faits et des complications de la vie quotidienne ne peuvent être considérés avec quelque pertinence comme concernant la justice sociale $»^{9}$. L'attention (le souci d'équité) se porte alors principalement sur l'organisation de la structure de base, qui doit être jugée d'un point de vue général. C'est un point de vue que l'on peut trouver adapté à une réalité sociale, que les agents abordent souvent en termes de récits biographiques individuels ou

\footnotetext{
${ }^{8}$ Voir J. Rawls, Théorie de la justice, p. 119.

${ }^{9}$ J. Rawls, Théorie de la justice, p. 119.
} 
d'histoires familiales (avec, donc, des substitutions des individus entre les positions sociales), plutôt qu'en termes de répartition instantanée entre des personnes données.

\subsection{Fragilité des échelles de valeur servant à repérer les inégalités.}

Il faut aussi essayer de comprendre, comme le soulignait R. Nozick, pourquoi certaines personnes préfèrent que d'autres n'aient pas de meilleurs résultats, dans quelle que dimension

que ce soit, en l'occurrence qu'elles soient à l'aise matériellement ou aient de la chance ${ }^{10}$. On se trouve alors confronté à une situation où une personne qui a un résultat, dans un domaine particulier, préférerait qu'un autre individu, ayant obtenu un résultat plus élevé $\mathrm{H}$, ait obtenu un résultat moins élevé que $\mathrm{H}$, même si cela n'élèvera pas son propre résultat. Ce cas montre que le sentiment qu'une personne a de sa propre situation ne dépend pas exclusivement de faits qui lui sont personnels: interviennent aussi dans cette évaluation des faits concernant d'autres personnes.

Considérons maintenant le problème d'une éventuelle dévalorisation de l'individu à ses propres yeux du fait de l'intériorisation de critères sociaux d'évaluation (" bon élève vs. mauvais élève », « bon ouvrier vs. mauvais ouvrier »). Comme le suggère Nozick, au profil factuel (ex : être capable de réussir au basket-ball quinze paniers sur 150 essais) s'ajoute un profil d'évaluation, permettant de représenter la façon dont chaque personne évalue ses propres scores sur le profil factuel. Il y aura aussi des classifications d'évaluations (par exemple excellent, bon, satisfaisant, médiocre, très mauvais), pour une personne donnée et pour chacune de ces dimensions. Ainsi dans une société où les gens s'accordent à penser que certaines dimensions sont généralement très importantes (et qu'il y a des différences dans la façon dont les agents répondent à ces dimensions) et où certaines institutions groupent publiquement des gens (selon leur place sur ces dimensions), ceux qui obtiennent un résultat peu élevé peuvent se sentir ravalés à un rang substantiellement inférieur dans une société hiérarchique.

Ainsi les personnes peuvent se juger elles-mêmes en fonction de la position qu'elles occupent sur les échelles correspondant aux dimensions les plus importantes, par lesquelles elles diffèrent les unes des autres. Moins il y a de dimensions, moins l'individu pourra adopter (avec succès)

\footnotetext{
${ }^{10}$ R. Nozick, Anarchie, Etat, Utopie, Paris, PUF, 1988, p. 295.
} 
une stratégie de pondération non uniforme, qui donnera un plus grand poids à une dimension sur laquelle il obtient un score élevé. De même, une société qui voudrait éviter de trop grandes différences d'évaluations entre ses membres pourrait n'avoir aucune pondération commune de ces dimensions. Dans ce cas, il y aurait une diversité de listes différentes de dimensions et de pondérations. De la sorte, chaque personne aurait plus de chances de trouver des dimensions, que certaines autres personnes trouveraient également importantes, et pour lesquelles elle obtiendrait des résultats raisonnablement bons. Elle aurait ainsi une estimation d'elle-même favorable, non idiosyncrasique. On peut se demander si une telle fragmentation de la pondération sociale commune doit être le fruit d'un effort centralisé, pour supprimer certaines dimensions importantes, ou bien résulter de la vie sociale spontanée.

La complexification ou l'enrichissement des valeurs sociales de référence peut intervenir de manière décentralisée, dans un processus de rivalité et d'innovation, par exemple, dans l'éducation. Dans l'éducation, les inégalités peuvent être assez facilement tolérées, s'il y a un processus d'émulation et d'innovation, qui assure la contestabilité des avantages, qui existent pour certains, à un certain moment, d'une manière qui peut bénéficier à ceux qui arrivent plus tard mais aussi à ceux qui sont déjà engagés dans le processus et qui ne sont pas a priori du côté «favorisé ». C'est certainement dans cette direction, qui suppose la reconnaissance sociale de la fragmentation des valeurs et des chances dans la vie, que l'on peut placer des espoirs de rupture avec les blocages d'une société figée, perçue de manière prédominante comme suscitant des obstacles devant les ambitions individuelles, au lieu d'offrir une multitude de supports différenciés à des ambitions elles-mêmes ramifiées.

2. Le risque de démagogie autour de l'égalité et la compétition politique autour de l'égalité.

\subsection{Les contingences de l'élaboration du discours public sur l'égalité.}

Peut-être ne faut-il pas sous-estimer, en les rangeant trop vite parmi les conceptions typiquement réactionnaires, les arguments sceptiques sur l'égalité. Un auteur comme F.A. von 
Hayek insiste sur le risque de dissimulation de causes particulières et d'intérêts partiels, derrière les revendications d'égalité ${ }^{11}$. Or il y a bien là un risque réel ${ }^{12}$.

Tel est par exemple le cas des comparaisons internationales de salaires entre professionnels d'un secteur, offrant un niveau de rémunération confortable. Ici, les revendications d'égalité masquent mal des arguments, qui relèvent plutôt de la négociation ou de la pression sur les pouvoirs publics : fuite des cerveaux, risque d'abandon du métier au profit d'autres carrières, etc. Un deuxième exemple est offert par les quotas et les filières d'accès privilégiées pour les écoles. Il s'agit là d'un moyen de faire parler de l'école et de faire monter sa cote, en faisant apparaître comme désirable l'entrée dans ladite école. En France, par exemple, cette stratégie est utilisée par des établissements qui adoptent un système de sélection à l'entrée. On voit se présenter sous l'apparence d'arguments d'égalité des chances de simples opérations de promotion sectorielle.

Certains arguments qui relèvent effectivement de la négociation plutôt que de l'argumentation éthique peuvent être convaincants et importants (p. ex. ceux qui concernent un salaire suffisant pour ne pas partir à l'étranger ou pour ne pas changer de métier ; un salaire suffisant pour ne pas avoir à faire un second métier en parallèlement à son activité principale ; un salaire suffisant pour que les fonctionnaires ne cèdent pas à la corruption, etc.). Mais de tels arguments, bien sûr, ne sont pas nécessairement convaincants en même temps en termes d'équité, quoiqu'ils prennent superficiellement cette forme, dans des argumentaires publics.

\subsection{Les effets réels du discours sur l'égalité des chances.}

Le discours sur l'égalité des chances a des effets pervers - que l'on rencontre très vite - dans la mesure où il sanctifie les inégalités : bien souvent, on part des privilèges tels qu'ils sont et l'on défend l'égalité d'accès à ces privilèges, sans considérer qu'ils sont en eux-mêmes problématiques. Or cette attitude de déférence envers une structure sociale existante peut engendrer des blocages et des contraintes arbitraires dans les carrières et les choix de vie des

\footnotetext{
${ }^{11}$ Voir F. Hayek, Law, Legislation and Liberty, Chicago, University of Chicago Press, 1973.

${ }^{12}$ De façon générale, on associe souvent l'égalitarisme au sentiment personnel d'envie, à partir d'une confusion de l'envie et de la jalousie.
} 
individus. Elle requiert en effet que l'on porte attention à l'égalité réelle d'accès à des privilèges que l'on considère par ailleurs, au moins tacitement, comme normaux ou allant de $\operatorname{soi}^{13}$.

Incontestablement certaines inégalités sont justifiées par des questions d'aptitudes (p.ex : les qualités requises pour faire des études au niveau master - mais peut-être pas pour faire des études secondaires générales). On peut également tenir compte des besoins de la nation (ce sont précisément ces derniers que des corps et écoles tels que les Ponts-et-Chaussées, l'ENA et l'Ecole spéciale militaire cherchent à satisfaire).

Or les inégalités induites exigent certainement que l'on réfléchisse au maintien, pour les autres personnes, des moyens de réaliser les mêmes projets, à un certain niveau de description au moins (par ex. entrer dans une profession mobilisant des aptitudes comparables mais dans le secteur privé, plutôt que dans le secteur public, et après avoir pu acquérir des aptitudes comparables). Faute de quoi l'on court le risque de justifier une société d'ordres ou de privilèges, avec des àcôtés, comme les blocages dans certaines filières d'étude, qui peuvent être dommageables en termes d'égalité des perspectives dans la vie mais aussi en termes de développement économique (si les individus ne peuvent pas faire des études dans le secteur qui les intéresse, on peut s'attendre à ce qu'ils soient moins créatifs ou motivés, dans leur début de carrière, dans la création d'entreprise ou dans la création de métiers adaptés à leurs aspirations et au contexte économique). Le problème le plus profond n'est peut-être pas tant la question souvent discutée de la reproduction sociale, dans les positions privilégiées (analysée notamment par P. Bourdieu), que celle de la perpétuation institutionnelle de mécanismes de blocage et de sélection, qui contrarient les aspirations et ambitions individuelles, et finalement le développement économique.

C'est alors le caractère ouvert des types de formation et des professions, qui joue un rôle prédominant davantage, par exemple, que le caractère socialement élitiste de certaines institutions. Si une personne veut étudier la philosophie, il n'est probablement pas décisif qu'elle ne soit pas admise à Oxford (faute de «patronage » suffisant) alors qu'elle peut étudier la même discipline, dans des conditions comparables dans une université « en briques rouges ». En effet,

\footnotetext{
${ }^{13}$ Cela a un potentiel critique vis-à-vis des titres protégés et exclusifs, qui ne se réfèrent pas aux compétences acquises, mais au lieu de formation p.ex. («ingénieur des ponts et chaussées», « allocataire moniteur normalien », etc.) ou qui introduisent des différences de statut qui ne sont liées ni à l'ancienneté ni à l'expérience ni à une différence de métier, p.ex. entre «administrateur civil» (énarque) et "attaché d'administration centrale» (non énarque) ; dans les deux cas, d'une manière qui n'est pas seulement «une remarque informative en passant» (comme : ancien interne de la faculté de médecine de Lyon...) mais qui a aussi un lien avec des aspects fonctionnels (comme les conditions de nomination, la carrière...).
} 
ces deux options permettent de réaliser le même projet de vie, à un certain niveau de description au moins, même si à un certain moment de l'histoire, l'une des deux universités a eu un avantage initial face à l'autre, en termes de réputation ou de moyens. Par ailleurs l'excellence est ici une position essentiellement contestable, au sens où rien n'interdit à une communauté d'étudiants et de savants, très loin d'Oxford, de s'efforcer à l'excellence d'une manière qui peut finalement aboutir à une position d'éminence (certains excellents collèges d'Oxford sont précisément issus, historiquement, de ce type de processus).

Les conditions de réplicabilité des études, d'accès ouvert aux professions et de récompense de l'effort, dans l'acquisition des compétences, sont certainement plus déterminantes que des inégalités vestigiales, liées à l'ancienneté ou au prestige des institutions, à des habitudes élitistes ou paternalistes de recrutement, dans des secteurs institutionnels limités, et qui, au fond, n'induisent pas de blocage décisif pour les individus qui n'en «bénéficient » pas (le bénéfice n'étant d'ailleurs qu'un bénéfice présumé à confirmer ex post $)^{14}$.

Ainsi il peut y avoir une différence dans les chances initiales de réalisation de certaines variantes des projets de vie, suite à la fréquentation d'un établissement plutôt qu'un autre (p.ex. s'occuper de l'administration de la recherche ou de la politique culturelle dans de grandes organisations internationales), mais l'important est que cela soit contestable et réversible. La réversibilité sera avérée par ex. si telle faculté parvient à démontrer son excellence (grâce aux enseignants et aux étudiants) dans un certain domaine, qui n'est pas très bien couvert par l'autre institution; ou bien encore, si elle fait la preuve de la bonne adaptation de sa formation à des circonstances ou innovations sociales qui, ex ante, n'étaient pas entièrement prévisibles.

En revanche - et c'est là que doit s'exercer la critique -, il y a des cas de discriminations effectifs, comme c'est le cas en Irlande du Nord par exemple, où le fait d'avoir fait ses études dans des écoles catholiques induit des blocages arbitraires, pour lesquels il n'y a pas de compensation possible. La critique de l'élitisme, telle qu'on la trouve chez P. Bourdieu, par exemple a souligné l'importance de ces blocages, dans l'accès à des choix de vie. A la lumière de ces exemples et de ces considérations, il apparaît que la problématique de l'égalité des chances

\footnotetext{
14 L'idée sous-jacente, ici, est que ce qui est réellement problématique socialement, c'est le blocage de perspectives individuelles ou familiales de développement d'aptitudes, d'accès aux études et de capacité d'accès sans garanti bien sûr - aux différentes professions. Lorsqu'il y a un blocage « dur » tel que peuvent en induire des systèmes de numerus clausus, de concours dans l'accès aux études, etc., alors il y a un problème réel pour l'égalité des chances.
} 
gagnerait à être reformulée en termes de blocages décisifs, concernant l'éducation, les choix professionnel, les plans de vie des individus. Les blocages considérés sont décisifs, au sens où ils sont éthiquement décisifs. Par là se trouve proposée une alternative à la résolution égalitariste, en termes de biens primaires ou de capabilités (capacités), que J. Rawls et A. Sen proposent en réponse à la question de l'égalité des chances, dans le but d'assurer à chacun le plus large éventail possible d'opportunités.

En somme, il faut déporter le centre de gravité des préoccupations concernant l'égalité des chances et donner la première place aux blocages induits par des institutions peu évolutives. Il importe également de contester la permanence des positions privilégiées au lieu de développer la mixité sociale dans l'accès (probabiliste) aux privilèges.

\section{Qu'est-ce que recouvre l'enthousiasme pour l'égalité ?}

\subsection{L'existence de discriminations vécues comme arbitraires.}

Les inégalités paraissent particulièrement préjudiciables lorsqu'elles induisent une relation de domination - entendue comme la soumission à une volonté arbitraire - et de subordination entre les individus et, par conséquent, lorsque de telles inégalités sont à l'origine d'une détention unilatérale du pouvoir de certains agents sur d'autres. C'est l'une des manières dont on explique que les inégalités économiques sont à rejeter: elles tendraient à engendrer des rapports de domination. De même, les inégalités dérivées de privilèges, accordés à certains groupes ou à certains types de travail, en raison de l'origine raciale, du sexe ou d'un handicap, induisent des blocages arbitraires ou une position de soumission, qui les rendent intolérables.

Peut-on défendre la même conclusion, lorsqu'il est fait référence à des circonstances assurément tout aussi contingentes, comme les circonstances familiales, en particulier les chances induites par le fait d'être né dans une famille riche et instruite ou dans une famille pauvre et moins instruite, ou les chances induites par le fait d'habiter dans une région bien dotée en établissements scolaires ? Il y a là en effet des éléments parfaitement arbitraires mais il ne faut peut-être pas sous-estimer la part d'arbitraire, qui entre aussi dans l'identification sociale des « chances dans la vie » qui sont désirables. En réalité, les histoires familiales, les traditions culturelles, les usages locaux et bien d'autres considérations peuvent faire apparaître comme 
désirables pour certains, ou pour certains groupes, des perspectives dans la vie qui le sont moins pour d'autres groupes. Par exemple, à niveau de vie égal, être admis en deuxième année de master puis en doctorat à la Sorbonne peut se voir attribuer une très grande valeur dans une famille intéressée, de manière prédominante, par le monde de la culture, et une valeur plus problématique dans une famille dont la culture et l'histoire sont tournées vers l'industrie.

On peut enfin considérer que la valeur (financière) attachée à certains métiers est bien souvent discutable, en particulier lorsqu'elle est le fruit des forces du marché ${ }^{15}$. Le souci de l'équité s'efface alors devant le jeu du marché. Cela pose un problème moral seulement dans la perspective particulière où le revenu est interprété comme un signe de la dignité des carrières ou des choix de vie, ce qui est très loin d'être uniformément reconnu (les milieux artistiques offrant de nombreux contre-exemples).

\subsection{La question des besoins ${ }^{16}$.}

Une société humaine doit assurer à chacun suffisamment de ressources pour mener une vie décente. Dans ce cas, on peut alors vouloir organiser des services publics assurant une redistribution élémentaire, en particulier des biens répondant à des besoins de base. Ces derniers se définissent comme des besoins prioritaires et fondamentaux, c'est-à-dire comme des besoins dont la satisfaction est urgente mais également comme des moyens polyvalents (all-purpose means), permettant aux personnes de poursuivre leurs projets de vie non pas en raison des buts qu'elles ont élu mais en tant qu'elles sont des membres de l'espèce humaine. Ainsi les besoins fondamentaux sont «ce dont nous avons besoin pour survivre, pour être en bonne santé, pour éviter la faim, pour vivre correctement $»^{17}$. Ces besoins répondent à ce dont nous avons besoin non pas pour ou en vue d'autre chose, en ce sens qu'ils nous sont requis en un sens absolu et non instrumental. Néanmoins leur satisfaction pose un problème de seuils ${ }^{18}$.

\footnotetext{
${ }^{15}$ Voir Anne Phillips, Which Inequalities Matter?, Cambridge, Polity Press, 2000, p. 69.

${ }^{16}$ Voir les travaux de Griffin et Sen sur ce point. Voir Social Science and Medecine, Presses Universitaires de Cambridge, 2005.

${ }^{17}$ J. Griffin, Well-being. Its Meaning, Measurement and Moral Signifiance, Oxford, Clarendon Press, 1986, p. 42.

${ }^{18}$ Ainsi l'identification des besoins premiers est problématique puisqu'il n'est pas certain que la question des besoins et des besoins premiers se pose de façon identique selon que l'ensemble des membres d'une société a ou non accès à l'eau potable, selon qu'il s'agit ou non d'une société industriellement avancée comme la nôtre.
} 


\subsection{L'absence de contraintes et de blocages arbitraires dans les vies de chacun.}

Les exigences, en matière de contraintes et de blocages arbitraires dans les vies individuelles, ont conduit Rawls a distingué le « système de la liberté naturelle » de l'égalité libérale. Dans le système de la liberté naturelle, la répartition initiale est déterminée par les dispositions implicites dans l'idée de « carrières ouvertes aux talents ». Ces dispositions exigent une égalité formelle des chances, c'est-à-dire que «tous aient au moins les mêmes droits (légaux) d'accès à toutes les positions sociales pourvues d'avantages $\gg{ }^{19}$. La répartition initiale des actifs et des capacités y est fortement influencée par des contingences naturelles et sociales, car l'égalité, ou la similarité, des conditions sociales n'y est recherchée que dans la mesure où elle préserve les institutions nécessaires. La répartition actuelle des revenus et de la richesse est l'effet cumulatif de répartitions antérieures des atouts et talents naturels, qui ont été ou non développés, comme de leur utilisation, favorisée ou non, dans le passé par des circonstances sociales et des contingences. En d'autres termes, le système de la liberté naturelle autorise que la répartition soit influencée de manière indue par des facteurs arbitraires, d'un point de vue moral.

Or la notion d'égalité libérale, proposée par Rawls, vise à corriger ce défaut, en ajoutant une condition supplémentaire à la condition d'ouverture des carrières aux talents, en l'occurrence un principe de juste (fair) égalité des chances. L'idée ici est que les positions ne doivent pas seulement être ouvertes à tous, en un sens formel, mais que tous devraient avoir une chance équitable (fair) d'y parvenir ${ }^{20}$. Autrement dit, les individus, qui sont au même niveau de talent et de capacité et qui ont le même désir de les utiliser, devraient avoir les mêmes perspectives de succès, indépendamment de leur position initiale dans le système social. Ainsi il devrait y avoir, dans tous les secteurs de la société, des perspectives à peu près égales de culture et de réalisation pour tous les individus, qui ont des motivations et des dons semblables ${ }^{21}$. Par là, se trouve atténuée l'influence des contingences sociales et du hasard naturel sur la répartition.

\footnotetext{
${ }^{19}$ J. Rawls, Théorie de la justice, Paris, Seuil, 1987, p. 103.

${ }^{20}$ J. Rawls, Théorie de la justice, p. 103.

${ }^{21}$ Voir H. Sidgwick dans The Methods of Ethics, Indianapolis/Cambridge, Hackett Publishing Company, p. 285,
} note 1. 
Rawls ajoute que pour y parvenir, il est nécessaire que les « dispositions du marché libre [soient] placées dans le cadre d'institutions politiques et légales qui règlent les courants principaux de la vie économique et qui préservent les conditions sociales nécessaires à la juste (fair) égalité des chances ». Il s'agit, notamment, d' " empêcher les accumulations excessives de propriété et de richesse et à maintenir des possibilités égales d'éducation pour tous » - qui justifie que l'on tienne compte de l'inégalité économique ${ }^{22}$.

Toutefois une justice égale ne signifie pas que la société doive contribuer pour chacun, dans une proportion égale, à la réalisation de la meilleure vie, dont il est individuellement capable ${ }^{23}$. Pour ce faire il faudrait, en effet, pouvoir estimer la valeur relative des projets de vie et posséder une mesure de ce que peut être une proportion égale, pour des individus ayant des conceptions différentes de leur bien.

\subsection{L'égalité dans le domaine politique.}

Une société qui reconnaît tous les adultes comme politiquement égaux reconnaît leur droit égal à voter et à se présenter à des élections. Elle reconnaît également, dans certaines limites, la légitimité de leurs points de vue. Néanmoins cette égalité n'a de sens qu'à condition qu'une certaine convergence des situations économiques existe. A. Phillips, par exemple, suggère que «l'engagement en faveur de l'égalité politique établit des limites en matière d'inégalités économiques $»^{24}$. En effet, les droits formels à la participation politique exigent que soit posée la question de savoir comment ces droits sont implémentés.

\subsection{Le respect social de soi et l'absence de mépris pour une culture ou un groupe ethnique.}

Il importe en effet que le respect dû à chacun le soit indépendamment de sa position sociale. Une forme fondamentale d'égalité est ici en jeu. Or l'application conséquente du principe de la juste égalité des chances exige de considérer les individus indépendamment de l'influence de leur

\footnotetext{
${ }^{22}$ J. Rawls, Théorie de la justice, Paris, Seuil, 1987, p. 104.

${ }^{23}$ Voir W.K. Frankena, "Some Beliefs about Justice », The Lindley Lecture, University of Kansas, 1966, p. 14

${ }^{24}$ A. Philips, Which Equalities Matter ?, Cambridge, Polity Press, 2000, p. 73.
} sq. 
position sociale ${ }^{25}$. Néanmoins on peut penser que, de façon générale, l'égalité de respect entre les groupes, dont les circonstances matérielles de vie sont fort divergentes, est difficile à garantir $a$ priori (par la législation en particulier).

Certains ont pu penser qu'une égalité de respect n'est pas possible sans une convergence des situations matérielles ${ }^{26}$. David Miller, par exemple, souligne que la conviction d'une égale valeur des personnes ainsi que la perception de l'égalité des statuts sont difficiles à entretenir, lorsqu'existent de trop grandes différences de richesse et de pauvreté, dans le mode de vie des individus. L'égalité des statuts est incompatible avec l'extrême pauvreté et l'extrême richesse ${ }^{27}$. Bien que l'égalité ne signifie pas l'identité ou la similitude, elle ne peut être compatible avec un trop grand déséquilibre des chances de vie, du pouvoir et du revenu ${ }^{28}$. En effet, qu'on le veuille ou non, il existe une relation entre le statut social et les conditions matérielles. L'observation empirique dans les pays occidentaux montre toutefois que la coexistence de personnes ayant des niveaux de vie très distincts peut se faire dans le respect réciproque. Cela est d'ailleurs favorisé par les expériences de vie commune : lors d'un scrutin électoral, à l'occasion d'une fête familiale ou locale ou lors de la fête de fin d'année de l'école élémentaire, les différences de statut social n’empêchent pas la fraternité ou le respect réciproque.

On peut se demander si la problématique du lien entre respect et richesse n'est pas dissymétrique, si elle ne concerne pas davantage, en réalité plutôt, l'extrême pauvreté que l'extrême richesse. Il y a effectivement quelque chose de très dérangeant dans le sort réservé à des personnes privées de domicile fixe, qui ne sont accueillies nulle part, mais la coexistence avec la grande richesse ne change rien à l'affaire. Elle n'allège ni n'aggrave le fardeau. Moralement, il y a une dissymétrie : on peut se demander s'il est approprié, socialement adéquat, ou éventuellement requis par des mécanismes incitatifs complexes, de laisser des personnages privilégiés mener une existence oisive et luxueuse (ce sont là des questions d'adéquation politique ou économique). Néanmoins tel n'est pas le type de question que pose le spectacle de

\footnotetext{
${ }^{25}$ Voir B.A.O. Williams, "The Idea of Equality», Philosophy, Politics and Society, P. Laslett et W. C. Runciman (éd.), Oxford, Blackwell, 1962, p. 125-129.

${ }^{26}$ A. Philips, Which Equalities Matter ?, Cambridge, Polity Press, 2000, p. 95.

${ }^{27}$ David Miller, «What Kind of Equality Should the Left Pursue? », in Jane Franklin (éd.), Equality, London, Institute for Public Policy Research, 1997, p. 94. Voir aussi A. Philips, Which Equalities Matter?, p. 130.

${ }^{28}$ Les riches et les pauvres ont également le droit de recourir à la loi pour protéger leur propriété privée, et partagent le même droit de dormir la nuit dehors. Néanmoins cette considération souligne l'inanité de considérer l'égalité comme le simple et seul fait de traiter les individus de la même façon. Cependant la référence à l'identité ou à la similitude (à l'égal traitement) demeure pertinente, lorsqu'il est question de culture, de religion, d'origine ethnique.
} 
l'extrême pauvreté. Les questions sont ici plutôt : est-il moralement défendable de ne rien faire institutionnellement pour changer la condition des personnes privées de domicile fixe (les personnes qui occupent la position rawlsienne du «plus mal loti » et qui sont affrontées, jusque dans les pays riches, à des conditions très problématiques de morbidité et de mortalité) ?

Une société, qui ferme les yeux sur l'extrême pauvreté ou qui tolère que certains types de talent soient arbitrairement rémunérés cent fois plus que d'autres, ne reconnaît pas une égale valeur (humaine) à ses citoyens. Par là même, elle entretient la tendance des citoyens à ne pas se considérer comme des égaux. Néanmoins il ne s'agit pas ici de dire qu'une stricte égalité est nécessaire pour défendre et justifier l'égale valeur des concitoyens. On veut plutôt souligner que l'égalité des citoyens ne peut se pérenniser, s'il existe une trop grande pauvreté assortie d'inaction persistante. Dans cette mesure, le souci pour le respect de soi-même et l'effort pour donner aux plus défavorisés l'assurance de leur propre valeur est fondamental, ce qui suppose de poser des limites aux formes de hiérarchie et aux degrés d'inégalité que la justice autorise ${ }^{29}$. Rawls suggère ainsi qu'on ne doit, par exemple, pas toujours distribuer les ressources éducatives, que ce soit en totalité ou en partie, en fonction de leur résultat selon des critères de productivité, mais aussi en fonction de leur valeur d'enrichissement de la vie sociale et personnelle des citoyens, y compris des plus défavorisés.

La mise en évidence d'inégalités qui méritent, plus que d'autres, d'être prises en compte et compensées suggère qu'il est pertinent de ne pas avoir un point de vue unifié sur l'inégalité. En effet, la question pratique n'est pas tant de savoir «ce qui ne va pas» que d'identifier les domaines où une correction doit être apportée. Ainsi on peut considérer que l'actuelle distribution des ressources économiques est arbitraire et injuste sans juger que son évolution soit une priorité. En revanche, lorsque ces inégalités économiques entravent l'égalité politique, la nécessité de remédier à cette situation (à ce qui « ne va pas ») se révèle urgente.

\section{Conclusion}

Au cours des décennies récentes, Amartya Sen a défendu avec force la thèse d'après laquelle toute forme d'éthique sociale recouvre, en dernière analyse, une certaine forme de recherche

\footnotetext{
${ }^{29}$ Voir J. Rawls, Théorie de la justice, p. 137.
} 
d'égalité. Cela fournit le motif d'une sorte de programme de recherche, dont on peut d'ailleurs estimer qu'il a été partiellement mené à bien: identifier, dans chaque doctrine particulière d'éthique sociale (fût-elle libertarienne ou méritocratique) une certaine conception sous-jacente de l'égalité. C'est admettre que la charge de la preuve doit toujours être attribuée aux doctrinaires qui prétendent s'écarter du principe d'égalité, et c'est aussi spécifier d'emblée que la défense doit prendre la forme d'une référence au principe d'égalité.

Il ne s'est pas agi pour nous de mettre en doute l'importance de la référence à l'égalité entre les hommes dans certaines régions de l'éthique sociale, dont nous pensons par ailleurs que ce sont des régions fondamentales. Toutefois, cela n'implique pas que la référence à l'égalité doive être déterminante dans toutes les régions de l'éthique sociale. De plus, il n'est pas évident que considérer l'égalité comme le principe suprême en tous domaines soit la meilleure voie à emprunter pour bien cerner l'importance spécifique de l'égalité et le juste poids à donner à ce type de considération.

Il nous est apparu nécessaire d'examiner d'un point de vue critique non pas seulement les tentatives pour dévier par rapport à l'égalité, mais aussi les références au principe d'égalité l'obligation même que l'on se fait de se référer à ce principe. Nous croyons avoir identifié au moins quelques raisons de fond à l'appui d'un tel examen : dans un contexte politique, il faut tenir compte des inconvénients qui peuvent être liés à la recherche de l'égalité ; la référence à des principes d'égalité peut servir des fins très variées et structurer des interactions menant à des résultats eux-mêmes très variés (qui peuvent n'avoir aucun rapport assignable avec le progrès moralement significatif vers quelque forme d'égalité que ce soit); la référence à l'égalité peut être motivée par des raisons morales mais aussi par des raisons qui ne sont pas de nature morale.

\section{Bibliographie}

Berlin, Isaiah, « Equality », repr. in Frederick A. Olafson (éd.), Justice and Social Policy, Englewood Cliffs, NJ, Prentice-Hall.

Frankena, W.K., « Some Beliefs about Justice », The Lindley Lecture, University of Kansas, 1966.

Griffin, James, Well-being. Its Meaning, Measurement and Moral Signifiance, Oxford, Clarendon Press, 1986.

Hayek, Friedrich A., Law, Legislation and Liberty, Chicago, University of Chicago Press, 1973. 
Miller, David, «What Kind of Equality Should the Left Pursue? », in Jane Franklin (éd.), Equality, London, Institute for Public Policy Research, 1997.

Nozick, Robert, Anarchie, Etat, Utopie, Paris, PUF, 1988.

Oakeshott, Michael, «Le rationalisme en politique », tr. fr. Olivier Sedeyn, Cités, 14 [2003], Paris, PUF ; initialement dans Cambridge Journal, I [1947]).

Phillips, Anne, Which Inequalities Matter ?, Cambridge, Polity Press, 2000.

Picavet, Emmanuel, John Rawls, Théorie de la justice, I, Paris, Ellipses, 2001.

Rawls, John, Théorie de la justice [1971], Paris, Seuil, 1987.

Sidgwick, Henri, The Methods of Ethics, Indianapolis/Cambridge, Hackett Publishing Company.

Williams, Bernard A.O., « The Idea of Equality », Philosophy, Politics and Society, P. Laslett et W. C. Runciman (éd.), Oxford, Blackwell, 1962.

Social Science and Medecine, Presses Universitaires de Cambridge, début 2005. 